\title{
Genetic Polymorphism of 17 Y-Chromosomal STR Loci in the "On To'rt Uriw" Tribes of Karakalpak Population
}

\author{
Kurganov Sardarkhodja Karimovich* \\ Laboratory of Forensic Biological Examination of Human DNA, Republican Centre of Forensic Expertise, Tashkent City, Chilanzar district, Chilonzarskiy
} reg., House 29, Republic of Uzbekistan

${ }^{*}$ Corresponding author: Kurganov Sardarkhodja Karimovich, Laboratory of Forensic Biological Examination of Human DNA, Republican Centre of Forensic Expertise, Republic of Uzbekistan, Tashkent city, Chilanzar district, Chilonzarskiy reg., House 29, Republic of Uzbekistan; E-mail: sardorbioinformatik@mail.ru

Received: March 09, 2021; Accepted: March 16, 2021; Published: April 01, 2021

\begin{abstract}
Haplotypes and allele frequencies for the 17 Y-chromosomal short tandem repeat (Y-STR) loci, DYS456, DYS389I, DYS390, DYS389II, DYS458, DYS19, DYS385a/b, DYS393, DYS391, DYS439, DYS635, DYS392, Y GATA H4, DYS437, DYS438 and DYS448 were determined in a sample of "On To'rt Uriw" tribes 100 unrelated Karakalpak males living in the regions of Xojeli (20), Taxiatosh (20), Shimbai (20), Konirat (20) and Moynaq (20) using the Y-filer PCR Amplification Kit (Thermo Fisher Scientific). The gene diversity was 0.9998 (standard error: 0.005). The haplotype diversity calculated from the 17 Y-STR loci was 0.9977 and the discrimination capacity was 0.8890 . The DYS385 locus showed the highest gene diversity value (0.8765), while the DYS391 locus showed the lowest gene diversity value (0.5033).
\end{abstract}

Keywords: DNA analysis, Y chromosome, Population data

\section{Introduction}

There have been few reports regarding genetic polymorphisms at the Y-STR loci in Karakalpak population [1-4]. The Karakalpaks are a heterogenous people, their appearance ranging from European to Mongoloid. The Karakalpaks as a whole are divided into two divisions, known as arts, the Qon'irat and the On To'rt Uriw. The term On To'rt Urıw, which means fourteen tribes, is somewhat misleading since the On To'rt Uriw are actually composed of just four tribes: the Qitay, Qipshaq, Keneges, and the Man'g'tt. Also, The four tribes of the On To'rt Uriw are also divided into clans: the Qitay into 12, the Q1pshaq into 13, the Keneges into 8, and the Man'g'it into 4. The difference between a tribe and a clan is defined by matrimonial alliances. The Karakalpaks practice exogamy. It is not possible to marry within one's own clan, but it is possible to marry into another clan within one's own tribe.

The world population of Karakalpaks is probably less than 600,000 , making them one of the smallest Turkic groups in Central Asia. About $80 \%$ live in the Autonomous Republic of Karakalpakstan, the remainder being mainly located in other parts of Uzbekistan and Kazakhstan. For comparison, the Qazaqs may number up to 14 million worldwide, of which only $81 \frac{1}{2}$ million (about $60 \%$ ) live in Kazakhstan. Surprisingly the Karakalpaks are not the dominant population of Karakalpakstan - they make up less than one third of the population and are just outnumbered by Uzbeks, some of whom have moved into southern Karakalpakstan from other parts of Uzbekistan in recent years. This study aimed to investigate the haplotypes and allele frequencies for the 17 Y-STR loci in population and establish forensic DNA database.

\section{Materials and Methods}

\section{Objects of the Research}

The subjects of the study were blood samples and dried saliva on sterile gauze tampons, selected from 100 individuals.

\section{DNA Extraction}

Genomic DNA was extracted from peripheral blood and dried saliva samples using the phenol-chloroform-isoamyl alcohol method.

\section{DNA Quantification}

After isolation, the quantity of genomic DNA of each sample was determined by quantitative real-time polymerase chain reaction (PCR) using the Quantifiler ${ }^{\text {Tx }}$ Human Male DNA Quantification kit (Thermo Fisher Scientific), which includes internal positive control to test for the presence of PCR inhibitors in the DNA extracts. Quantitative realtime PCR was performed on 7500 Real-Time PCR System (Applied Biosystems).

\section{PCR Amplification and Detection}

To ensure successful amplification, $0.5 \mathrm{ng}$ to $1 \mathrm{ng}$ of DNA was used for each multiplex amplification reaction. All thermal cycling was conducted on Applied Biosystems ${ }^{\circ}$ GeneAmp ${ }^{\circ}$ PCR System 9700 thermal cyclers. PCR amplification using Y-filer PCR Amplification 
Kurganov Sardarkhodja Karimovich (2021) Genetic Polymorphism of 17 Y-Chromosomal STR Loci in the "On To'rt Urıw" Tribes of Karakalpak Population.

Table 1:

\begin{tabular}{|c|c|c|c|c|c|c|c|c|c|c|c|c|c|c|c|c|}
\hline № & 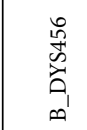 & $\begin{array}{l}\vec{a} \\
\infty \\
\tilde{n} \\
\hat{0} \\
\infty^{\prime}\end{array}$ & 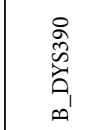 & 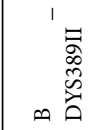 & 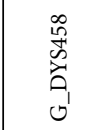 & $\begin{array}{l}\stackrel{a}{\vec{n}} \\
\underset{0}{0} \\
0\end{array}$ & $\begin{array}{l}m \\
\tilde{N} \\
\tilde{D}_{1} \\
\nu_{1}\end{array}$ & $\begin{array}{l}\vec{\sigma} \\
\hat{\tilde{n}} \\
\overline{0}_{1} \\
\lambda_{1}\end{array}$ & 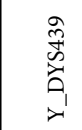 & 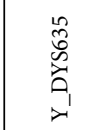 & 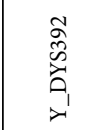 & 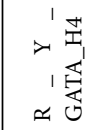 & 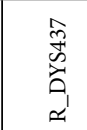 & 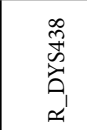 & 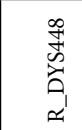 & $\begin{array}{l}\infty \\
\infty \\
\tilde{D}_{0}^{\infty} \\
0 \\
0\end{array}$ \\
\hline 8 & & & & & & & & & & & & & & 0,0108 & & 0,0430 \\
\hline 9 & & & & & & & & 0,0753 & & & & 0,0323 & & 0,0968 & & 0,0376 \\
\hline 10 & & & & & & & & 0,6667 & 0,3118 & & 0,0215 & 0,0215 & & 0,3656 & & 0,0108 \\
\hline 11 & & & & & & & & 0,2258 & 0,3333 & & 0,5914 & 0,4409 & & 0,4624 & & 0,1505 \\
\hline 12 & & 0,1828 & & & & & 0,2903 & 0,0323 & 0,2473 & & 0,0538 & 0,4301 & & 0,0645 & & 0,1774 \\
\hline 13 & & 0,5591 & & & & 0,0645 & 0,6129 & & 0,0538 & & 0,1398 & 0,0753 & 0,0215 & & & 0,2097 \\
\hline 14 & 0,0968 & 0,2581 & & & 0,0215 & 0,3118 & 0,0968 & & 0,0538 & & 0,1720 & & 0,6452 & & & 0,1452 \\
\hline 15 & 0,5269 & & & & 0,1075 & 0,3333 & & & & & 0,0108 & & 0,2151 & & & 0,0645 \\
\hline 16 & 0,2473 & & & & 0,2473 & 0,2151 & & & & & 0,0108 & & 0,1183 & & & 0,0376 \\
\hline 17 & 0,0968 & & & & 0,3656 & 0,0753 & & & & & & & & & & 0,0538 \\
\hline 18 & 0,0323 & & & & 0,1398 & & & & & & & & & & 0,0860 & 0,0376 \\
\hline 19 & & & 0,0430 & & 0,0860 & & & & & 0,0215 & & & & & 0,2366 & 0,0054 \\
\hline 20 & & & & & 0,0323 & & & & & 0,0645 & & & & & 0,4839 & 0,0215 \\
\hline 21 & & & & & & & & & & 0,2688 & & & & & 0,0645 & 0,0054 \\
\hline 22 & & & 0,0968 & & & & & & & 0,1505 & & & & & 0,1290 & \\
\hline 23 & & & 0,2581 & & & & & & & 0,2903 & & & & & & \\
\hline 24 & & & 0,2473 & & & & & & & 0,1828 & & & & & & \\
\hline 25 & & & 0,3441 & & & & & & & 0,0215 & & & & & & \\
\hline 27 & & & 0,0108 & 0,0215 & & & & & & & & & & & & \\
\hline 28 & & & & 0,1183 & & & & & & & & & & & & \\
\hline 29 & & & & 0,3978 & & & & & & & & & & & & \\
\hline 30 & & & & 0,2258 & & & & & & & & & & & & \\
\hline 31 & & & & 0,1613 & & & & & & & & & & & & \\
\hline 32 & & & & 0,0753 & & & & & & & & & & & & \\
\hline $\begin{array}{l}\text { G e n e } \\
\text { diversity } \\
\text { (D) }\end{array}$ & 0,6484 & 0,5937 & 0,7506 & 0,7527 & 0,7735 & 0,7436 & 0,5365 & 0,5033 & 0,7326 & 0,7908 & 0,6040 & 0,6201 & 0,5288 & 0,6459 & 0,6891 & 0,8765 \\
\hline
\end{tabular}

Table 2:

\begin{tabular}{|c|c|c|c|c|c|c|c|c|c|c|c|c|c|c|c|c|}
\hline ID & DYS456 & DYS389I & DYS390 & DYS389II & DYS458 & DYS19 & DYS385 & DYS393 & DYS391 & DYS439 & DYS635 & DYS392 & YGATAH4 & DYS437 & DYS438 & DYS448 \\
\hline Sample 1_Xojeli & 17 & 13 & 25 & 29 & 16 & 16 & 11,13 & 13 & 10 & 11 & 23 & 11 & 12 & 14 & 11 & 20 \\
\hline Sample 2_Xojeli & 15 & 13 & 23 & 32 & 16 & 13 & 15,17 & $14 \mathrm{~A}$ & 10 & 13 & 22 & 14 & 11 & 13 & 11 & 19 \\
\hline Sample 3_Xojeli & 15 & 14 & 19 & 30 & 17 & 14 & 13,13 & 13 & 10 & 13 & 24 & 13 & 11 & 15 & 10 & 19 \\
\hline Sample 4_Xojeli & 15 & 13 & 25 & 31 & 16 & 15 & 11,15 & 13 & 11 & 10 & 23 & 11 & 12 & 14 & 11 & 20 \\
\hline Sample 5_Xojeli & 15 & 13 & 25 & 31 & 16 & 15 & 11,15 & 13 & 11 & 10 & 23 & 11 & 12 & 14 & 11 & 20 \\
\hline Sample 6_Xojeli & 15 & 13 & 22 & 29 & 19 & 15 & 9,16 & 12 & 10 & 12 & 21 & 14 & 11 & 16 & 10 & 18 \\
\hline Sample 7_Xojeli & 15 & 13 & 24 & 29 & 18 & 14 & 13,18 & 12 & 10 & 11 & 20 & 14 & 12 & 15 & 11 & 20 \\
\hline Sample 8_Xojeli & 15 & 12 & 24 & 27 & 17 & 14 & 11,14 & 12 & 11 & 13 & 23 & 14 & 11 & 15 & 12 & 19 \\
\hline Sample 9_Xojeli & 16 & 14 & 24 & 30 & 17 & 15 & 11,14 & 13 & 10 & 11 & 23 & 13 & 12 & 14 & 11 & 18 \\
\hline Sample 10_Xojeli & 14 & 14 & 23 & 30 & 17 & 14 & 12,13 & 14 & 10 & 10 & 22 & 14 & 12 & 14 & 10 & 19 \\
\hline Sample 11_Xojeli & 16 & 14 & 24 & 30 & 17 & 15 & 11,14 & 13 & 10 & 11 & 23 & 13 & 12 & 14 & 11 & 18 \\
\hline Sample 12_Xojeli & 15 & 13 & 25 & 29 & 18 & 17 & 12,12 & 13 & 10 & 10 & 21 & 11 & 11 & 14 & 10 & 22 \\
\hline Sample 13_Xojeli & 15 & 12 & 23 & 28 & 19 & 15 & 13,18 & 12 & 10 & 12 & 22 & 11 & 11 & 15 & 9 & 22 \\
\hline Sample 14_Xojeli & 14 & 13 & 23 & 29 & 17 & 14 & 11,13 & 14 & 10 & 10 & 22 & 14 & 12 & 14 & 10 & 19 \\
\hline
\end{tabular}




\begin{tabular}{|c|c|c|c|c|c|c|c|c|c|c|c|c|c|c|c|c|}
\hline Sample 15_Xojeli & 16 & 14 & 24 & 29 & 16 & 15 & 12,15 & 12 & 10 & 14 & 24 & 14 & 13 & 14 & 12 & 18 \\
\hline Sample 16_Xojeli & 15 & 13 & 25 & 29 & 17 & 17 & 12,13 & 13 & 10 & 10 & 21 & 11 & 11 & 14 & 10 & 22 \\
\hline Sample 17_Xojeli & 15 & 13 & 25 & 31 & 15 & 16 & 11,14 & 13 & 11 & 10 & 24 & 11 & 12 & 14 & 11 & 20 \\
\hline Sample 18_Xojeli & 15 & 13 & 25 & 29 & 17 & 17 & 12,12 & 13 & 10 & 10 & 21 & 11 & 11 & 14 & 10 & 21 \\
\hline Sample 19_Xojeli & 15 & 13 & 25 & 29 & 17 & 17 & 12,12 & 13 & 10 & 10 & 21 & 11 & 11 & 14 & 10 & 21 \\
\hline Sample 20_Xojeli & 15 & 14 & 23 & 30 & 15 & 15 & 11,21 & 14 & 10 & 11 & 21 & 11 & 11 & 14 & 10 & 21 \\
\hline Sample 1_Taxiatosh & 15 & 13 & 22 & 30 & 17 & 14 & 13,13 & 12 & 10 & 11 & 24 & 11 & 11 & 15 & 9 & 20 \\
\hline Sample 2_Taxiatosh & 18 & 12 & 22 & 29 & 16 & 15 & 13,13 & 12 & 10 & 11 & 22 & 11 & 11 & 14 & 12 & 20 \\
\hline Sample 3_Taxiatosh & 15 & 12 & 24 & 29 & 20 & 14 & 13,18 & 12 & 10 & 13 & 19 & 14 & 12 & 16 & 11 & 20 \\
\hline Sample 4_Taxiatosh & 16 & 13 & 23 & 28 & 17 & 14 & 11,14 & 13 & 11 & 11 & 23 & 13 & 12 & 15 & 12 & 19 \\
\hline Sample 5_Taxiatosh & 15 & 13 & 23 & 29 & 18 & 14 & 12,18 & 14 & 10 & 10 & 24 & 10 & 12 & 16 & 11 & 19 \\
\hline Sample 6_Taxiatosh & 15 & 13 & 24 & 29 & 17 & 14 & 13,18 & 12 & 10 & 11 & 20 & 14 & 12 & 15 & 11 & 20 \\
\hline Sample 7_Taxiatosh & 15 & 13 & 25 & 29 & 18 & 16 & 12,13 & 13 & 10 & 10 & 22 & 11 & 11 & 14 & 10 & 22 \\
\hline Sample 8_Taxiatosh & 15 & 13 & 22 & 30 & 15 & 14 & 13,13 & 13 & 11 & 13 & 23 & 13 & 11 & 15 & 10 & 20 \\
\hline Sample 9_Taxiatosh & 16 & 14 & 24 & 30 & 17 & 15 & 11,14 & 13 & 10 & 11 & 23 & 13 & 12 & 14 & 11 & 18 \\
\hline Sample 10_Taxiatosh & 14 & 12 & 23 & 28 & 16 & 14 & 13,15 & 13 & 10 & 11 & 21 & 11 & 11 & 16 & 10 & 20 \\
\hline Sample 11_Taxiatosh & 16 & 14 & 25 & 32 & 15 & 15 & 11,12 & 13 & 11 & 10 & 23 & 11 & 14 & 14 & 11 & 18 \\
\hline Sample 12_Taxiatosh & 15 & 13 & 22 & 29 & 18 & 17 & 9,17 & 12 & 10 & 12 & 21 & 14 & 11 & 16 & 10 & 19 \\
\hline Sample 13_Taxiatosh & 14 & 13 & 22 & 31 & 19 & 14 & 13,15 & 12 & 10 & 12 & 22 & 11 & 11 & 16 & 10 & 19 \\
\hline Sample 14_Taxiatosh & 14 & 13 & 22 & 31 & 19 & 14 & 13,15 & 12 & 10 & 12 & 22 & 11 & 11 & 16 & 10 & 19 \\
\hline Sample 15_Taxiatosh & 15 & 13 & 24 & 30 & 20 & 14 & 13,16 & 12 & 10 & 11 & 21 & 11 & 11 & 15 & 10 & 19 \\
\hline Sample 16_Taxiatosh & 15 & 14 & 24 & 32 & 14 & 16 & 11,12 & 13 & 9 & 10 & 23 & 11 & 12 & 14 & 11 & 20 \\
\hline Sample 17_Taxiatosh & 16 & 13 & 23 & 29 & 16 & 14 & 12,14 & 13 & 10 & 11 & 21 & 15 & 12 & 14 & 10 & 19 \\
\hline Sample 18_Taxiatosh & 18 & 13 & 23 & 31 & 16 & 15 & 13,13 & 12 & 10 & 12 & 22 & 11 & 11 & 16 & 9 & 20 \\
\hline Sample 19_Taxiatosh & 14 & 14 & 23 & 30 & 17 & 14 & 11,13 & 15 & 10 & 10 & 22 & 16 & 11 & 14 & 11 & 19 \\
\hline Sample 20_Taxiatosh & 16 & 13 & 25 & 29 & 16 & 16 & 11,14 & 13 & 10 & 11 & 23 & 11 & 12 & 14 & 11 & 20 \\
\hline Sample 1_Shimbai & 15 & 14 & 23 & 31 & 17 & 14 & $15.2,15.2$ & 12 & 10 & 12 & 24 & 11 & 11 & 14 & 9 & 21 \\
\hline Sample 2_Shimbai & 15 & 12 & 25 & 29 & 18 & 16 & 11,14 & 13 & 11 & 10 & 23 & 11 & 13 & 14 & 11 & 20 \\
\hline Sample 3_Shimbai & 15 & 13 & 25 & 29 & 18 & 16 & 12,13 & 13 & 10 & 12 & 21 & 11 & 11 & 14 & 10 & 22 \\
\hline Sample 4_Shimbai & 14 & 12 & 24 & 27 & 17 & 17 & 11,14 & 13 & 10 & 12 & 21 & 11 & 12 & 15 & 11 & 20 \\
\hline Sample 5_Shimbai & 15 & 12 & 23 & 29 & 17 & 13 & $17.2,17.2$ & 13 & 10 & 12 & 21 & 11 & 9 & 14 & 10 & 20 \\
\hline Sample 6_Shimbai & 15 & 12 & 23 & 29 & 18 & 15 & 12,12 & 12 & 10 & 12 & 24 & 12 & 12 & 14 & 11 & 20 \\
\hline Sample 7_Shimbai & 15 & 13 & 24 & 29 & 18 & 15 & 12,12 & 13 & 10 & 11 & 21 & 11 & 11 & 14 & 10 & 22 \\
\hline Sample 8_Shimbai & 15 & 13 & 25 & 29 & 17 & 17 & 12,12 & 13 & 10 & 10 & 21 & 11 & 11 & 14 & 10 & 22 \\
\hline Sample 9_Shimbai & 16 & 13 & 25 & 30 & 15 & 15 & 11,14 & 13 & 11 & 10 & 24 & 11 & 12 & 14 & 11 & 20 \\
\hline Sample 10_Shimbai & 15 & 13 & 24 & 29 & 19 & 15 & 12,12 & 13 & 10 & 11 & 21 & 11 & 11 & 14 & 10 & 22 \\
\hline Sample 11_Shimbai & 16 & 13 & 24 & 32 & 16 & 15 & 11,15 & 12 & 10 & 10 & 24 & 11 & 12 & 14 & 11 & 20 \\
\hline Sample 12_Shimbai & 16 & 13 & 24 & 32 & 16 & 15 & 11,15 & 12 & 10 & 10 & 24 & 11 & 12 & 14 & 11 & 20 \\
\hline Sample 13_Shimbai & 17 & 13 & 27 & 31 & 15 & 15 & 11,14 & 13 & 12 & 11 & 23 & 11 & 11 & 14 & 11 & 20 \\
\hline Sample 14_Shimbai & 15 & 13 & 25 & 31 & 16 & 15 & 11,15 & 13 & 11 & 10 & 23 & 11 & 12 & 14 & 11 & 20 \\
\hline Sample 15_Shimbai & 15 & 12 & 23 & 29 & 17 & 13 & 16,17 & 13 & 10 & 12 & 21 & 11 & 9 & 14 & 10 & 20 \\
\hline Sample 16_Shimbai & 16 & 14 & 25 & 30 & 16 & 15 & 11,11 & 13 & 10 & 12 & 21 & 7 & 11 & 14 & 11 & 19 \\
\hline Sample 17_Shimbai & 18 & 13 & 22 & 31 & 16 & 15 & 13,13 & 12 & 10 & 12 & 22 & 11 & 11 & 16 & 9 & 20 \\
\hline Sample 18_Shimbai & 15 & 12 & 24 & 28 & 17 & 16 & 12,19 & 12 & 10 & 11 & 22 & 13 & 11 & 15 & 10 & 19 \\
\hline Sample 19_Shimbai & 14 & 14 & 25 & 31 & 16 & 15 & 12,14 & 13 & 11 & 10 & 23 & 14 & 11 & 14 & 11 & 20 \\
\hline Sample 20_Shimbai & 15 & 13 & 25 & 31 & 16 & 15 & 11,15 & 13 & 11 & 10 & 23 & 11 & 12 & 14 & 11 & 20 \\
\hline Sample 1_Konirat & 15 & 13 & 25 & 29 & 18 & 16 & 12,13 & 13 & 10 & 10 & 21 & 11 & 11 & 14 & 10 & 22 \\
\hline
\end{tabular}




\begin{tabular}{|c|c|c|c|c|c|c|c|c|c|c|c|c|c|c|c|c|}
\hline Sample 2_Konirat & 16 & 14 & 23 & 30 & 17 & 14 & 11,12 & 13 & 11 & 12 & 24 & 14 & 12 & 15 & 12 & 20 \\
\hline Sample 3_Konirat & 15 & 14 & 19 & 30 & 17 & 14 & 13,13 & 13 & 10 & 14 & 24 & 13 & 11 & 15 & 10 & 19 \\
\hline Sample 4_Konirat & 16 & 13 & 25 & 29 & 17 & 16 & 11,14 & 13 & 11 & 10 & 23 & 11 & 13 & 14 & 11 & 20 \\
\hline Sample 5_Konirat & 16 & 13 & 25 & 31 & 15 & 17 & 11,13 & 13 & 10 & 11 & 23 & 11 & 13 & 14 & 11 & 20 \\
\hline Sample 6_Konirat & 15 & 13 & 24 & 28 & 15 & 15 & 13,17 & 13 & 10 & 11 & 21 & 13 & 12 & 14 & 10 & 18 \\
\hline Sample 7_Konirat & 15 & 12 & 24 & 28 & 19 & 15 & 11,16 & 12 & 10 & 12 & 20 & 12 & 12 & 15 & 10 & 19 \\
\hline Sample 8_Konirat & 16 & 13 & 25 & 30 & 19 & 16 & 11,14 & 13 & 11 & 10 & 23 & 11 & 12 & 14 & 11 & 20 \\
\hline Sample 9_Konirat & 15 & 12 & 22 & 28 & 17 & 15 & 12,15 & 14 & 10 & 11 & 21 & 11 & 10 & 16 & 10 & 22 \\
\hline Sample 10_Konirat & 15 & 14 & 24 & 30 & 18 & 13 & 13,14 & 13 & 9 & 10 & 21 & 11 & 12 & 14 & 10 & 20 \\
\hline Sample 11_Konirat & 17 & 13 & 23 & 28 & 19 & 13 & 13,16 & 13 & 10 & 11 & 23 & 16 & 11 & 14 & 11 & 22 \\
\hline Sample 12_Konirat & 16 & 14 & 25 & 32 & 16 & 15 & 11,14 & 13 & 10 & 10 & 23 & 12 & 11 & 14 & 11 & 20 \\
\hline Sample 13_Konirat & 15 & 14 & 23 & 31 & 17 & 14 & $15.2,15.2$ & 12 & 10 & 12 & 24 & 11 & 11 & 14 & 9 & 21 \\
\hline Sample 14_Konirat & 15 & 12 & 24 & 28 & 17 & 16 & 12,19 & 12 & 10 & 11 & 22 & 13 & 11 & 15 & 10 & 19 \\
\hline Sample 15_Konirat & 16 & 13 & 25 & 31 & 17 & 16 & 12,14 & 13 & 11 & 11 & 24 & 11 & 13 & 14 & 11 & 20 \\
\hline Sample 16_Konirat & 16 & 13 & 23 & 30 & 16 & 13 & 15,17 & 14 & 11 & 12 & 23 & 14 & 11 & 13 & 11 & 19 \\
\hline Sample 17_Konirat & 15 & 13 & 24 & 29 & 16 & 14 & 13,17 & 12 & 10 & 12 & 21 & 11 & 11 & 14 & 10 & 20 \\
\hline Sample 18_Konirat & 15 & 13 & 23 & 29 & 17 & 14 & 13,18 & 12 & 10 & 11 & 20 & 14 & 12 & 15 & 11 & 20 \\
\hline Sample 19_Konirat & 16 & 13 & 25 & 29 & 16 & 15 & 13,14 & 13 & 10 & 12 & 20 & 11 & 12 & 14 & 11 & 20 \\
\hline Sample 20_Konirat & 17 & 14 & 25 & 32 & 15 & 16 & 11,14 & 13 & 11 & 10 & 23 & 11 & 12 & 14 & 11 & 21 \\
\hline Sample 1_Moynaq & 15 & 12 & 24 & 28 & 18 & 14 & $12.2,12.2$ & 13 & 10 & 12 & 20 & 14 & 12 & 15 & 11 & 20 \\
\hline Sample 2_Moynaq & 17 & 13 & 23 & 28 & 19 & 13 & 13,16 & 13 & 10 & 11 & 23 & 16 & 11 & 14 & 11 & 22 \\
\hline Sample 3_Moynaq & 14 & 13 & 23 & 28 & 15 & 14 & 13,16 & 12 & 9 & 11 & 21 & 11 & 13 & 15 & 9 & 20 \\
\hline Sample 4_Moynaq & 15 & 14 & 19 & 30 & 17 & 14 & 13,14 & 13 & 11 & 14 & 24 & 13 & 11 & 15 & 10 & 19 \\
\hline Sample 5_Moynaq & 15 & 14 & 19 & 30 & 17 & 14 & 13,13 & 13 & 10 & 14 & 24 & 13 & 11 & 15 & 10 & 19 \\
\hline Sample 6_Moynaq & 17 & 13 & 25 & 29 & 16 & 16 & 11,14 & 13 & 10 & 14 & 23 & 11 & 12 & 14 & 11 & 20 \\
\hline Sample 7_Moynaq & 17 & 12 & 25 & 29 & 17 & 16 & 10,14 & 13 & 11 & 11 & 23 & 11 & 12 & 14 & 11 & 21 \\
\hline Sample 8_Moynaq & 16 & 13 & 23 & 29 & 18 & 14 & 12,18 & 14 & 10 & 10 & 24 & 10 & 12 & 16 & 11 & 19 \\
\hline Sample 9_Moynaq & 16 & 13 & 25 & 29 & 17 & 16 & 11,14 & 13 & 12 & 10 & 23 & 11 & 13 & 14 & 11 & 20 \\
\hline Sample 10_Moynaq & 15 & 13 & 22 & 28 & 19 & 16 & 9,17 & 12 & 10 & 11 & 22 & 13 & 11 & 16 & 10 & 18 \\
\hline Sample 11_Moynaq & 15 & 12 & 23 & 30 & 17 & 15 & 12,17 & 12 & 10 & 12 & 19 & 13 & 12 & 15 & 10 & 19 \\
\hline Sample 12_Moynaq & 17 & 13 & 25 & 29 & 16 & 16 & 11,14 & 13 & 10 & 11 & 23 & 11 & 12 & 14 & 11 & 20 \\
\hline Sample 13_Moynaq & 16 & 14 & 25 & 32 & 16 & 15 & 11,14 & 13 & 10 & 10 & 23 & 12 & 11 & 14 & 11 & 20 \\
\hline Sample 14_Moynaq & 15 & 14 & 24 & 31 & 20 & 15 & 12,12 & 13 & 9 & 11 & 22 & 11 & 10 & 14 & 10 & 20 \\
\hline Sample 15_Moynaq & 14 & 14 & 23 & 30 & 16 & 14 & 11,13 & 14 & 11 & 10 & 22 & 14 & 12 & 14 & 10 & 19 \\
\hline Sample 16_Moynaq & 17 & 12 & 24 & 29 & 17 & 15 & 12,14 & 13 & 10 & 12 & 21 & 11 & 11 & 14 & 11 & 20 \\
\hline Sample 17_Moynaq & 15 & 13 & 24 & 29 & 14 & 14 & 13,17 & 12 & 10 & 12 & 21 & 11 & 11 & 14 & 10 & 20 \\
\hline Sample 18_Moynaq & 16 & 13 & 25 & 31 & 17 & 16 & 12,12 & 13 & 11 & 11 & 24 & 11 & 12 & 14 & 11 & 20 \\
\hline Sample 19_Moynaq & 17 & 13 & 25 & 29 & 16 & 16 & 11,14 & 13 & 10 & 11 & 23 & 11 & 12 & 14 & 11 & 20 \\
\hline Sample 20_Moynaq & 17 & 12 & 25 & 29 & 17 & 16 & 10,14 & 13 & 11 & 11 & 23 & 11 & 12 & 14 & 11 & 21 \\
\hline
\end{tabular}

Kit (Thermo Fisher Scientific) was performed as recommended by the manufacturer, although half of the recommended reaction volume $(12.5 \mu \mathrm{l})$ was used.

Separation and detection of the 17 Y-STR loci were performed using the 3130xl Genetic Analyser (Applied Biosystems) 16-capillary array system and filter set G5. Each sample was prepared by adding $1 \mathrm{~mL}$ PCR product to $14 \mathrm{~mL}$ of $\mathrm{Hi}-\mathrm{Di}^{\mathrm{mix}}$ formamide and $0.4 \mathrm{~mL}$ GeneScanTM-500 LIZ $^{\text {rx }}$ internal size standard (Thermo Fisher Scientific).
The sample run data were analyzed, together with an allelic ladder and positive and negative controls, using GeneMapper ID-X v3.2 (Applied Biosystems) software.

\section{Statistical Analysis}

Comparison information of the sample data was generated using an in-house software program involving DNA-expert macros designed to check for allele sharing across all loci. For all analyses the DYS385 
locus was treated as a single haplotype and not two separate alleles. The gene diversity $(\mathrm{D})$ was calculated as $\mathrm{D}=\frac{n}{n-1}\left(1-\sum p_{1}^{2}\right)$, where $p_{1}$ is

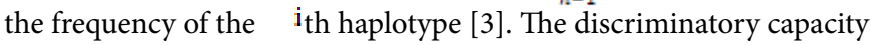
was determined by dividing the number of different haplotypes by the number of samples in that population. The discrimination capacity (DC) was determined by the formula $\mathrm{n} / \mathrm{N}$ where $\mathrm{n}=$ the number of observed haplotypes divided by the number of samples [1].

\section{Results and Discussion}

This population was demonstrated 100 haplotypes, of which 93 were unique. The gene diversity was 0.9998 (standard error: 0.005). The haplotype diversity calculated from the 17 Y-STR loci was 0.9977 and the discrimination capacity was 0.8890 . The DYS385 locus showed the highest gene diversity value $(0.8765)$, while the DYS391 locus showed the lowest gene diversity value $(0.5033)$.

\section{Funding}

This work was supported by Ministry of Innovation Development of The Republic of Uzbekistan, Grant No. A-2-089+(A-2-056).

\section{References}

1. Coble MD, Hill CR, Butler JM (2013) Haplotype data for 23 Y-chromosome markers in four US population groups. Forensic Sci Int Genet 7: 66-68. [crossref]

2. Kurganov S, Axmedova D, Filatova V, Muxamedov R, Axmedov B (2018) Genetic Polymorphisms at 17 Y-STR loci in Uzbek Population. Peer Re J Foren \& Gen Sci.

3. Nei M, Tajima F (1981) DNA polymorphism detectable by restriction endonucleases. Genetics 97: 145-163. [crossref]

4. Raphaelle Chaix, Fre'de'ric Austerlitz, Tatyana Khegay, Svetlana Jacquesson, Michael F. Hammer, et al. (2004) The Genetic or Mythical Ancestry of Descent Groups: Lessons from the Y Chromosome. Am J Hum Genet 75:1113-1116. [crossref] 